\title{
Multicentre Clinical Trial Monitoring
}

\section{Tamilselvi Srinivas* and Ankanagari Srinivas}

Quest Life Sciences Pvt Ltd, SDF III, MEPZ, Tambaram, Chennai, India

\begin{abstract}
A standard quality control plan was established to monitor the quality conductance at Multicentre trials. Case report forms were designed; qualified investigators, and study personnel were trained on given protocol. Monitoring personnel who appointed for the trial has experience with monitoring program. It ensures overall guaranteed data accuracy and to prevent or to identify protocol deviations/violations. Clinical site, physicians and study personnel were audited, and the study conductance processes were audited as well. In addition, the forms of the case reports are reviewed for completeness and internal consistency. The eligibility and validity of the patients was verified in this study, and the data was monitored for compliance and accuracy. Supplementing and improving the existed procedures for quality monitoring will ensure that multi-centre clinical trials are considered as valid and scientifically stringent. This paper provides practical approach for implementing strict monitoring plan for randomized controlled trials (RCTs).
\end{abstract}

\section{Keywords: Multicentre clinical trial; Quality; Monitoring}

Abbreviations: CT: clinical trial; MCT: Multicenter clinical trial; REC: Research ethics committee

\section{Introduction}

To conduct a multi-center clinical trial with high quality, the personnel have to be trained on especially GCP, protocol, SOP and other applicable regulatory requirements to compete with international standard. These trials are performed according to the principles of the Declaration of Helsinki (Version Edinburgh 2000). And the trial protocols have been approved by local institutional review board and ethics committee.

\section{Methods}

The practice of quality monitoring in multicentre clinical trials focuses on making the standard quality control plan. This includes the design of case report form (CRF) and the training of study personnel. The duty of these monitors is to guarantee data accuracy and to prevent or detect protocol violations such as enrollment of non eligible patients based on inclusion and exclusion criteria or assignment of improper treatment. Therefore, the monitors should check the following requirements: medical background, training in GCP, and having relevant experience of clinical trials; familiar with trial protocol and standard operating procedures monitoring the whole procedures, including filling in the Case Report Forms as well as modification error; taking adequate time to do the monitoring work periodically according to the plan.

During Recruitment: Initially monitor shall check the recruitment is done as per the protocol within the agreed recruitment period," by the investigator and whether the investigators have sufficient time to conduct and complete the trial within the agreed trial period" [1]. Information regarding recruitment per site is included in the Multi centre clinical trial (MCT) final report when reporting trials results to regulatory agencies [2]. Check for any new recruiting centers or investigators are added to the original list. And the records of agreement between all the parties are available in the site [3]. Since all the parties involved needs to have transparency in CTs [4]. MCT is registered in publicly available clinical trial registry in India and it provides detailed information about the names, contact details, recruitment commitments, and recruitment delivers of all site principal investigators involved. The same information is available to the sponsor, participating patients and recruiting investigators, or any other interested in the field [5].

\section{Preparation work on quality monitoring}

The monitoring plan includes: deciding the number of monitors in each clinical center, planning the supervision speed in terms of clinical trial progress; adjusting the inspection frequency based on the trial schedule. The monitoring assessment forms includes all the activities from screening to post study follow up and study completion as per the GCP regulations, and these forms are designed according to the features of clinical trial.

Implementation is the main aspect of the monitoring procedure, which includes the following: reading the study summaries, feedback comments and on-the-spot suggestions from each clinical center before the inspections, maintaining a good communication with the principal investigator of each centre, clinicians, patients and laboratory examiners, to be informed of every concrete flow of procedure and the progress; taking notes about the problems, flaws, and on-the-spot photos; summarizing the monitoring results and evaluate the integrity and credibility of clinical data collections [6].

\section{Quality monitoring}

At clinical site: At clinical site, the resources including instruments ready for study use and are having valid calibration certificates and its traceability certificates for its working performance. And the laboratory which involves for sample analysis should have NABL or CAP accreditations are in line with the requirements of protocol and SOPs. Meanwhile, the monitors should also examine the training record and curriculum vitae of clinical investigators, and other study personnel whether all of them are well trained as per the clinical study requirement/sponsor requirement.

Trial procedures: The clinician's play an important role and their duties are recruiting, enrolling, screening, randomized allocation,

*Corresponding author: Tamilselvi Srinivas, Quest Life Sciences Pvt Ltd, SDF III, MEPZ, Tambaram, Chennai 600 045, India, E-mail: manickamtamilselvis@ gmail.com, asrinivasmail@yahoo.co.in

Received October 03, 2012; Accepted October 19, 2012; Published October 21 2012

Citation: Srinivas T, Srinivas A (2012) Multicentre Clinical Trial Monitoring Pharmaceut Reg Affairs S12:002. doi:10.4172/2167-7689.S12-002

Copyright: $\odot 2012$ Srinivas T, et al. This is an open-access article distributed under the terms of the Creative Commons Attribution License, which permits unrestricted use, distribution, and reproduction in any medium, provided the original author and source are credited. 
treating patients and follow-up. Therefore, it is indispensable to ensure that the clinicians grasp the procedures systematically.

Monitors inspect the center-randomization list and check the clinicians have confirmed the case histories and laboratory tests that meet inclusion criteria. And also check whether the clinicians have done the dosing as per randomization schedule given by the sponsor. Check whether the diagnosis, disease stages and classifications conform to the inclusion criteria; use the patient's medical records, the corresponding conclusions of laboratory examinations, as well as the records of patients with symptoms, to determine the certainty of basic information of illness. Inspect whether or not the course of the treatment strictly corresponds to the randomized implementation of the corresponding treatment measures including the selection criteria and SOPs adherence, etc. Check all procedures are recorded includes by taking pictures.

A special attention is paid to the physicians' explanations on whether are they following the clinical research requirements as per DGCI and the GCP regulation during communication with the subjects during the trial.

CRFs for completeness and internal consistency: In order to verify the validity, integrity, and accuracy of trial documents, monitors have to check thoroughly all the CRFs.

Patients' eligibility and validity: Monitoring personnel shall verify the signed informed consent by the subjects, and randomly check the patients contact phone number provided on the CRF, so as to ensure the authenticity of the subjects, and to check the diagnosis and the main treatment experiences. At the same time, monitors review the original medical histories and laboratory tests in order to confirm the patients' eligibility.

\section{Data monitoring}

Monitors ensure the data recording in CRFs are compliant with the medical records and laboratory reports. In particular, the monitors need to review records for the key elements as follows:

Check the testing time of samples, the contents of laboratory tests, and the filling time in forms to guarantee the timeliness and integrity. Some randomly selected subjects were asked on-the-spot regarding their case histories, treatments and drug combination in order to audit the facility and completeness of CRF documents. The monitors specifically checks past history including the information about the drug usage before patients' enrollment and whether patients combine the medication therapy during the trial. Moreover, if the patient took any drugs, the clinicians should record the information in the CRF form with the start-stops date. However, if the patients took the medication that is not allowed, clinician evaluates the subject whether fit to continue or not. The dosage of medicine, the time of taking the medicine, the time of pain relief and the side effects of the medicine needs to be recorded chronologically.

Adverse events: Sponsors and monitors pay special attention on the recording and verification of safety monitoring and adverse events. Any adverse events must be recorded includes entire chronology of the event. All of the adverse events must be handled and followed up. All the patients in the trial need to complete the post study follow up, in order to assess and compare the results in different treatment group. Monitors have to inspect study personnel are documenting the followup, through telephone.

Subjects dropout and lost to follow-up: The quantities of dropouts, suspension and lost to follow-up, are assessed for its authenticity. Check the details of withdrawn if any. The causes of withdrawn commonly have the following conditions: Patients return to home town and they cannot return follow up treatment and patients violate advice and refuse to comply with the protocol. In this case, doctors should arouse patients' initiative, improve the attitude in clinics, and exhibit more concerns in order to obtain patients' trust and cooperation, and strengthen their compliance.

\section{Results}

\section{Evaluating the source documents}

Each clinical site should have site master file, including patients' records, laboratory checking reports, Updated CRF forms and other essential documents. Monitors evaluate whether there is omission or loss in relevant materials and reports.

After assessing of on-site performance, monitors have to accomplish the monitoring report. The report is submitted to regulatory, and the sponsors. All case history reports must be completed in handwriting. The monitors put forward recommendations and suggestions to all the sites involved. The contents of monitoring report should focus on review of regulatory requirements, $\mathrm{CRO}$ audit, recommendations for improving performance from the $\mathrm{CRO}$ and sponsor audit and if any recommendations from previous audits. Regular meetings should be arranged to discuss improvements in monitoring standards.

During the subject enrollment, if any lab parameter found to be abnormal, such as the ECG test or platelet counts, but they did not have any correlated history or pathological symptoms, clinicians has to ensure the same as with in normal condition before subject check in. During the clinical study process, clinicians have to enhance communication with patients and often inquire the concomitant therapy comprehensively during the treatment process.

\section{Supervising the trials}

During the initial stage of clinical trials, monitor and other study personnel will take part in the GCP and protocol training, in order to have knowledge of GCP and completely ensure the implementation of the same during the clinical trial process, and participate themselves in quality trials. Monitor responsibilities are not only the clinical trial monitoring, but facilitating collaboration among study coordinators and sponsors. During the audit schedule, the practical difficulties are avoided through informal discussions, with other site study personnel, in case of any difficulty shall contact the sponsor to coordinate.

The large sample, multi-center clinical trials have high-quality requirements in inclusion criteria as per the protocol. During treating course and follow-up period clinicians have to pay much attention to details. Monitoring personnel give them timely information regarding the progress of the trial, in order to know the importance and necessity of their work.

\section{Conclusion}

Large randomized controlled trials addressing the effectiveness of treatment, Monitoring work in these multi-centre clinical trials are essential for data accuracy and the evaluation of study objectives in clinical trials. Review all of the source documents and source data, and assess whether the coordinators carry out these researches under the requirement of GCP with an objective, rigorous, careful, and scientific attitude. Quality assurance is very crucial in the conductance of clinical trial. Implementing the regulatory requirements for repeated quality monitoring will ensure concurrent data entry and considered as valid data. 


\section{Acknowledgements}

We gratefully acknowledge the Quest Lifesciences Pvt Ltd for their support in preparing and publishing this article.

\section{References}

1. (2010) International Conference on Harmonisation of technical requirements for Registration of Pharmaceuticals for Human Use ICH Harmonised Tripartite Guideline. Guideline for Good Clinical Practice.

2. European Medicines Agency (2010) Clinical trials submitted in marketing authorization applications to the EMA. Overview of patient recruitment and geographical location of investigator sites.

3. National Institutes of Health (2011) ClinicalTrials.gov

4. Food and Drug Administration Amendments Act of 2007.

5. Zarin DA, Tse T, Williams RJ, Califf RM, Ide NC (2011) The ClinicalTrials.gov results database—update and key issues. N Engl J Med 364: 852-860.

6. Guthrie LB, Oken E, Sterne JAC, Gillman MW, Patel R, et al. (2012) Ongoing monitoring of data clustering in multicenter studies. BMC Medical Research Methodology 12: 29 